S. Kwok, M. Dopita, and R. Sutherland, eds.

\title{
Far-Infrared Atomic Lines: PDRs or Shocks?
}

\author{
Margaret Meixner, David Fong \& Edmund C. Sutton \\ University of Illinois, Dept. of Astronomy, MC-221, Urbana, IL 61801
}

Arancha Castro-Carrizo \& Valentín Bujarrabal

Observatorio Astronómico Nacional, Apartado 1143, E-28800, Alcalá de Henares, Spain

William B. Latter

Caltech, SIRTF Science Center, MS 314-6, Pasadena, CA 91125

Alexander Tielens

Kapteyn Astronomical Inst. Postbus, 800, Groningen, 9700 Netherlands

Douglas M. Kelly

Univ. of Arizona, Steward Obs., Tucson, AZ, 85721

Michael J. Barlow

Department of Physics and Astronomy, University College London, Gower Street, London WC1E 6BT, United Kingdom

\section{Introduction}

The relative role of the stellar radiation field, the stellar outflows and the interstellar radiation field (ISRF) in transforming the molecular ejecta into atomic gas was the subject of our ISO LWS and SWS spectroscopy study of 24 evolved stars which span the range from AGB stars to proto-planetary nebulae (PPNs) and PNs. The far-infrared (FIR) atomic fine-structure lines are powerful probes of the warm atomic gas in photodissociation regions (PDRs) and shocks. This paper summarizes and compares the ISO spectroscopy studies of carbon-rich (C-rich) and oxygen-rich (O-rich) evolved stars, published by Fong et al. (2001) and Castro-Carrizo et al. (2001), respectively. We find that photodissociation, not shocks, is responsible for the chemical change from molecular to atomic gas.

\section{ISO Results}

The HR diagram (Figure 2) shows our sample and some results. Besides unusual sources, like Betelgeuse, which has an active chromosphere, and Mira, which has a hot companion, AGB stars are undetected in these lines which means that the ISRF is not an important contributor to the observed FIR atomic lines. In fact, we do not start detecting these lines until the central star reaches a temperature 


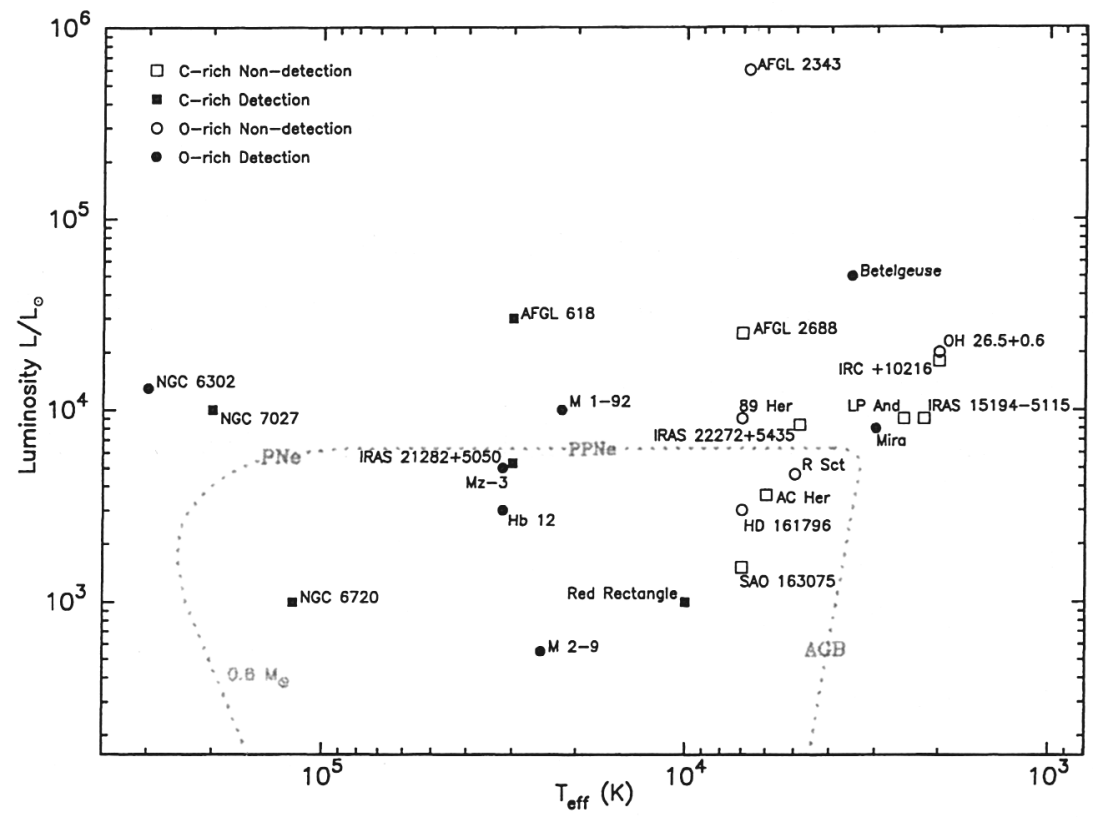

Figure 1. Objects from this ISO study plotted on the HR diagram.

of $10000 \mathrm{~K}$ (the Red Rectangle). All stars with higher effective temperatures are detected. At effective temperatures higher than $10000 \mathrm{~K}$, we find that the intensity of the [OI] $63 \mu \mathrm{m}$ line, which is the brightest line, increases and we also start detecting other lines such as [CII] $158 \mu \mathrm{m}$, [OI] $146 \mu \mathrm{m}$ and [SiII] 35 $\mu \mathrm{m}$. Such a trend is expected for PDRs because higher $\mathrm{T}_{\text {eff }}$ means that more starlight comes out in the FUV which is responsible for creating and heating the PDR.

Figure 2 plots the line widths of the FIR atomic lines against the CO line widths for seven objects. If the FIR atomic lines arise in PDRs they should have comparable line widths to the $\mathrm{CO}$ lines whereas if these FIR lines arise in shocked-gas regions their line widths should be significantly larger. The black line represents a one-to-one correspondence for the line widths and the fact that most of the data cluster around this line indicates a PDR origin. The one outlier source, AFGL 618, has both a narrow line component arising from a PDR and a broad line component probably arising from shocked gas.

Comparison of the FIR line intensities with PDR and shocked-gas models also supports the interpretation that PDRs dominate the chemical evolution. Figure 3 compares the observed line intensities with the C-rich and O-rich PDR models which have very different chemistries. Note that higher gas densities (n) and higher incident FUV radiation (G) produces higher line intensities. The [CII] line intensities for the C-rich models are higher than O-rich models as expected for higher carbon abundance, but the [OI] line intensities are comparable for the two models because C-rich PDRs tend to be hotter. The observed line intensities fall within the parameter space of the PDR models and specific obser- 


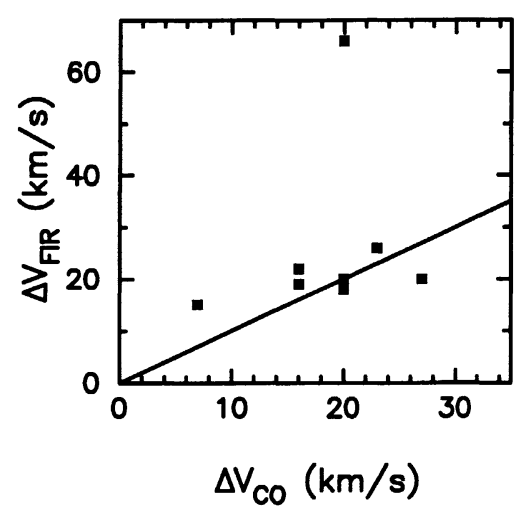

Figure 2. Comparison of FIR FWHM line widths with the CO FWHM line widths.

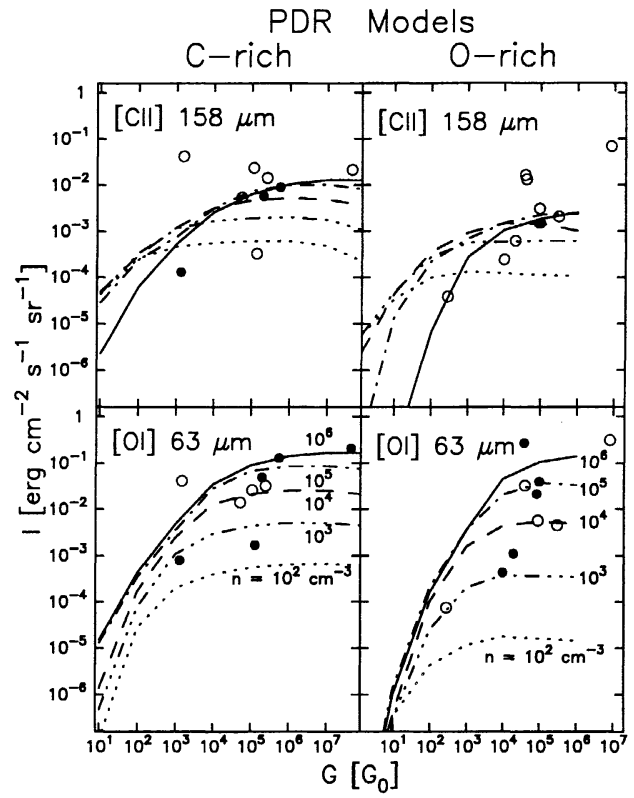

Figure 3. Comparison of the observed fine-structure atomic lines with PDR models. The curves represent the predicted intensities for models with different densities, $\mathrm{n}$. $\mathrm{G}$ is a measure of the incident farUV radiation field in units of the ISRF $\left(\mathrm{G}_{0}\right)$. The filled circles indicate detections and the open circles indicate upper limits along the y-axis. 
vations fit the models well. Comparison of the FIR line intensities with J- and C- shocked-gas models is considerably worse. C-shocks are ruled out because of the presence ionized species such as [CII]. J-shocks are ruled out because the [CII] $158 \mu \mathrm{m}$ line intensities are too high.

\section{Atomic gas masses}

We can estimate the amount of neutral atomic mass from the [CII] $158 \mu \mathrm{m}$ line intensity using the following formula,

$$
\mathrm{M}_{\text {atomic }}\left(\mathrm{M}_{\odot}\right) \sim 0.1\left(\frac{\mathrm{F}_{\mathrm{CII}}}{2.1 \times 10^{-11} \mathrm{erg} \mathrm{cm}^{-2} \mathrm{~s}^{-1}}\right)\left(\frac{\mathrm{D}}{1 \mathrm{kpc}}\right)^{2}\left(\frac{\mathrm{F}_{\text {cor }}}{1}\right)\left(\frac{1.5 \times 10^{-3}}{\mathrm{X}_{\mathrm{c}}}\right)
$$

where $\mathrm{F}_{\mathrm{CII}}$ is the observed $[\mathrm{CII}]$ flux, $\mathrm{D}$ is the distance, $\mathrm{F}_{\text {cor }}$ is the correction factor which is greater than 1 when [CII] is not thermalized and $X_{\mathrm{C}}$ is the carbon abundance which is $3 \times 10^{-4}$ for C-rich sources and $1.5 \times 10^{-3}$ for O-rich sources. In Table 1, we list the mass estimates for our sources detected in the [CII] line. The sources are ordered from least to most evolved. The third column shows the fractional neutral atomic mass where $M_{\text {total }}=M_{\text {atomic }}+M_{\text {ionized }}+M_{\text {molecular }}$. The fourth column shows the fractional atomic, both neutral and ionized, mass. For the C-rich sources, the fractional atomic gas mass increases with evolutionary stage as expected for the expanding influence of a PDR. Interestingly, for the O-rich sources, the atomic gas mass fraction is nearly 1.0 for all three sources independent of evolutionary stage. If these results hold for a larger sample of objects, then it would suggest that the gas in O-rich sources is almost instantly photodissociated when the $\mathrm{T}_{\text {eff }}$ of the star is hot enough.

\begin{tabular}{|c|c|c|c|}
\hline Source & $\mathrm{M}_{\text {atomic }}\left(\mathrm{M}_{\odot}\right)$ & $\frac{\mathrm{M}_{\text {atomic }}}{M_{\text {total }}}$ & $\frac{M_{\text {atomic }}+M_{\text {ionized }}}{M_{\text {total }}}$ \\
\hline C-rich & & & \\
\hline AFGL 618 & 0.01 & 0.006 & 0.006 \\
\hline IRAS $21282+5050$ & 0.1 & 0.04 & 0.04 \\
\hline NGC 7027 & 0.2 & 0.12 & 0.15 \\
\hline NGC 6720 & 0.06 & 0.1 & 0.4 \\
\hline O-rich & & & \\
\hline M 2-9 & 0.04 & 0.8 & 0.9 \\
\hline $\mathrm{Hb} 12$ & 0.3 & 0.95 & 1.0 \\
\hline NGC 6302 & 1.3 & 0.81 & 0.94 \\
\hline
\end{tabular}

\section{References}

Castro-Carrizo, A., Bujarrabal, V., Fong, D., Meixner, M., Tielens, A.G.G.M., Latter, W.B. \& Barlow, M.J. 2001, A\&A, 367, 674

Fong, D., Meixner, M., Castro-Carrizo, A., Bujarrabal, V., Latter, W.B., Tielens, A.G.G.M., Kelly, D.M., \& Sutton, E.C. 2001, A\&A, 367, 652 